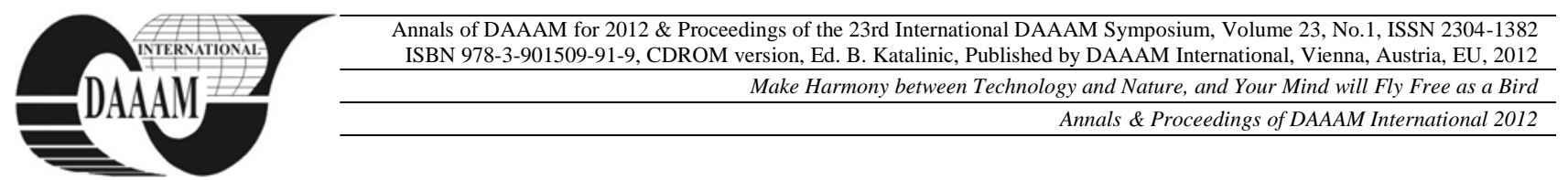

\title{
MULTI-BAND FILTER BUILT WITH A PERIODICALLY PATTERNED STRIPLINE
}

\author{
DE SABATA, A[ldo]; MATEKOVITS, L[adislau]; SILAGHI, H[elga] M[aria]; ROHDE, U[lrich] L. \& \\ SILAGHI, A[lexandru] M[arius]
}

\begin{abstract}
A novel unit cell consisting of a meander shaped metallic patch, positioned in between two dielectric grounded layers and connected to one of them by three vias is proposed. The large number of reactive elements composing the structure are shown to determine an equally large number of electromagnetic band-gaps making the structure appropriate for advanced sensor or multi-band filtering applications in modern wireless systems. Parametric studies considering the value of the dielectric constant of one of the dielectric layers, for two orthogonal directions of propagation are reported.
\end{abstract}

Keywords: strip-line, patterned surfaces, electromagnetic bandgaps, filtering, sensors

\section{INTRODUCTION}

Microstrip boards can be used as devices able to conduct or to prevent propagation of surface Bloch waves when a metallic periodic pattern is impressed on one face, opposed to the ground plane [1]. Slow waves, having phase velocities smaller than the speed of light propagate along the surface, while fast waves are radiated away, allowing construction of antennas with precisely controlled directions of radiations, or even steerable [2]. Furthermore, another particularity of such a surface consists of the existence of one or several electromagnetic band-gaps (EBGs), which are contiguous frequency ranges where signals are prevented to propagate along the surface. Moreover, the surface presents high impedance for an impinging electromagnetic wave and acts like an artificial magnetic conductor [1].

The metal patches etched on the surface of the board can be connected to the ground plane through one or several vias. The number and position of the vias influences the performances of the EBG structure, providing flexibility in design if such a structure is imposed by the requirements of a particular situation, such as multi-band filtering for wireless applications [3] or parallel-plate bouncing noise suppression in high speed digital or mixed-signal circuits $[4,5,6]$.

A further modification consists of covering the structure with a metal plane at a certain distance above the patterned surface. This solution prevents leakage of the electromagnetic field, since it is reflected by the metallic cover; however, EBGs continue to exist in between different frequency limits - if vias are present (parallel-plate modes propagate at any frequency in the absence of vias) [7]. A second dielectric layer separates the patterned plane from the upper metal plane, in the same way as the original one separates it from the ground plane. The dielectric constants of the two layers can be equal or different in value. The resulting device, having the structure of an inhomogeneous strip-line, is more compact than the open structure, is non-radiating and finds applications in filtering, antennas feeders and parallel-plate noise suppression [3-7] just to mention some. In modern wireless communications systems, the existence of a large number of EBGs, whose positions in frequency can be technologically controlled, is an important issue [3]. Another important subject in view of dispersion engineering applications is the mono-modal propagation in between EBGs. This opens possibilities to conception of new devices with remarkable properties such as super-lens, super-prisms and propagation controllers [8, 9]. A structure with a large number of EBGs (up to 8) between every two modes of propagation has been recently reported in [10].

Filtering is a natural application of EBG materials due to the band-structure. Multiband and broad-band filters in microstrip and suspended strip-line technology are current subject of research (see e.g. [11]). In microstrip filter design, patterns introduced as defects in the ground plane are known as a solution for frequency response shaping [12].

A non-uniform series of circular slots or annular rings in the ground plane parallel to the signal line of a microstrip board, with dimensions proportional to coefficients of binomial and Chebyshev polynomials, have been shown to provide low passband ripples and distinct stop-bands [13].

A repeated pattern of resonant loads etched in the ground plate below a microstrip line has been proposed as a 1D structure of filter inspired from EBG materials [14]. A design methodology, including control of ripples in pass-bands has been devised.

In this communication, a structure consisting of a patterned surface included between two dielectric layers and two metal planes is considered in view of filtering applications. The periodic pattern is based on a square unit cell containing a meander-type metal patch, connected to the ground plane by three vias. It is expected for such a structure to have many resonant frequencies and, consequently, several EBGs. The wave propagation related properties of the proposed device are assessed via dispersion diagrams (DDs) [15] calculated by means of a commercial solver [16]. Calculation of DDs forces one to consider an infinite extent of the surface. However, in a practical situation, the device 



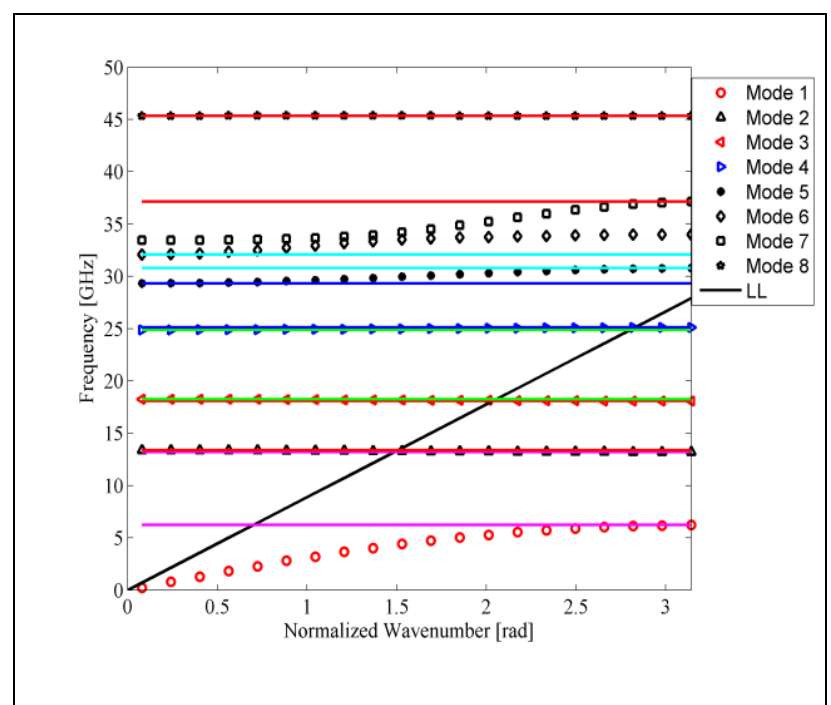

Fig. 3. DD for propagation in the $y$ direction, $\gamma_{12}=15$

The DD for $x$-propagating waves with $\gamma_{r 2}=100$ (a socalled "high $k$ material" [5]) is reported in Fig. 2, and the DD for $y$-propagating waves in the case of, $\gamma_{r 2}=15$ is reported in Fig. 3. Light lines (LLs), corresponding to propagation of waves in the stratified dielectric medium (without the patterned surfaces) and separating slow and fast waves [4] are also represented in both figures.

The DD in Fig. 2 reveals existence of EBGs between every two consecutive modes. However, not all these EBGs are present for lower values of the dielectric constant of the upper dielectric layer. An example involving the other direction, $y$, is reported in Fig. 3, where no EBG is present between modes 6 and 7 . Nevertheless, the palette of EBGs has resulted quite large in all cases, due to resonances that are determined by the reactive elements, such as the capacitive nature of the patch with respect to the ground, the stray capacitance between patches, the inductance of vias and nonetheless the mutual coupling between the constitutive elements of the patch; the structure behaves like coupled transmission lines.

In most cases, the structure exhibits resonant modes, like modes 2-5 in Fig. 2 and modes 2-4 and 8 in Fig. 3. Advantage can be taken of this feature by incorporating the proposed device in the structures of sensors with several observables [20].

In connection to the result in Fig. 3, it is noteworthy the large guard-bands that separate the resonant modes from the rest of the propagating modes. This feature makes the structure particularly useful for dispersionengineering applications.

In a given design situation, it is important to be able to adapt the structure to meet particular requirements. The proposed geometry offers the possibility of several geometric and material parameters to be varied in order to change the positions of the EBGs. In the present communication, results of varying the dielectric constant of the upper dielectric layer $\gamma_{r 2}$ between 1 (free space) and 15 are reported for waves propagating in the two principal, orthogonal directions.

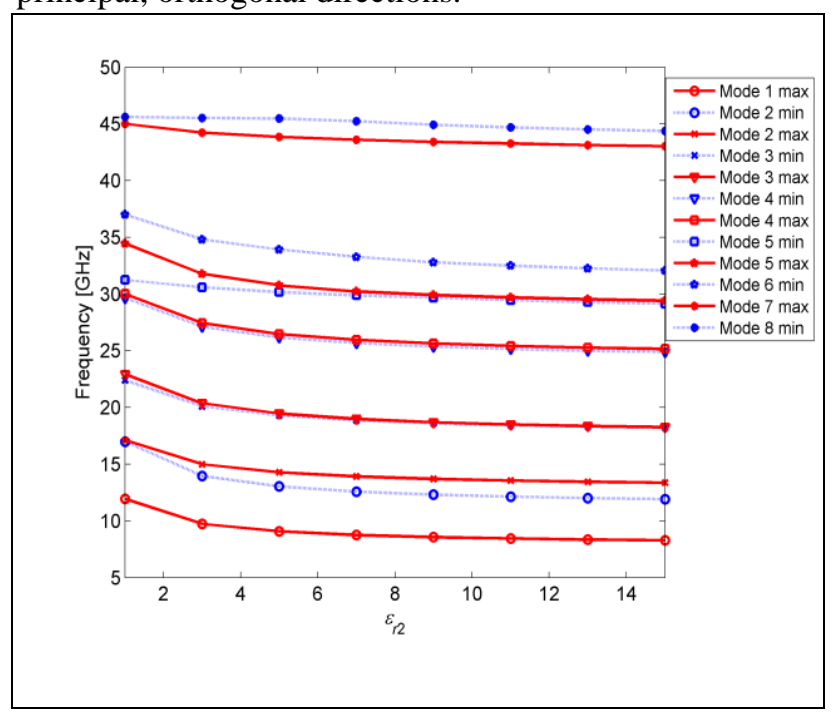

Fig. 4. Frequency limits of the EBGs versus the dielectric constant of the upper dielectric layer for $x$-directed waves

Several other values of $\gamma_{r 2}$ have also been considered, but are not reported here because of space limitations. The width of an EBG is considered to be defined by the maximum frequency of the lower mode that borders from below the EBG and the minimum frequency of the upper bordering mode. In Fig. 4, the limit frequencies of the EBG for waves propagating in the $x$ direction are reported; six EBGs are present for all values of the dielectric constant in the considered range, in this case. On the figures, the EBGs extend between curves marked similarly.

The limit frequencies for waves propagating in the $y$ direction are presented in Fig. 5. Up to $\gamma_{r 2}=3$, five EBGs are present, after which the number increases to six. Such a characteristic can be explained by the trapped electromagnetic field in the higher dielectric constant media. A resonant mode is present whenever two consecutively, differently marked, represented curves are very close. The values of the frequency-related parameters that define the EBGs decrease when the dielectric constant increases, as expected. Since the variation is moderate, the values of these parameters are conveniently controllable and do not resent the variation with respect to the nominal value.

The resonant behavior of modes 3 and 4 as displayed in Fig. 4 extends for the whole considered range of the dielectric constant, while mode 5 is resonant for roughly half of that range. This feature is important since conservation of the qualitative properties helps adapting the structure for various requirements concerning frequency. The same is true for modes 2,3 and 4 as displayed in Fig. 5.

In performing the parametric study of the influence of the dielectric constant on the DD we have considered only small values of this quantity since in every-day applications it is supposed that low-cost materials are preferred. However, as Fig. 2 reveals, use of "high $k$ " 
materials may add new and useful features that might be interesting for some specific applications.

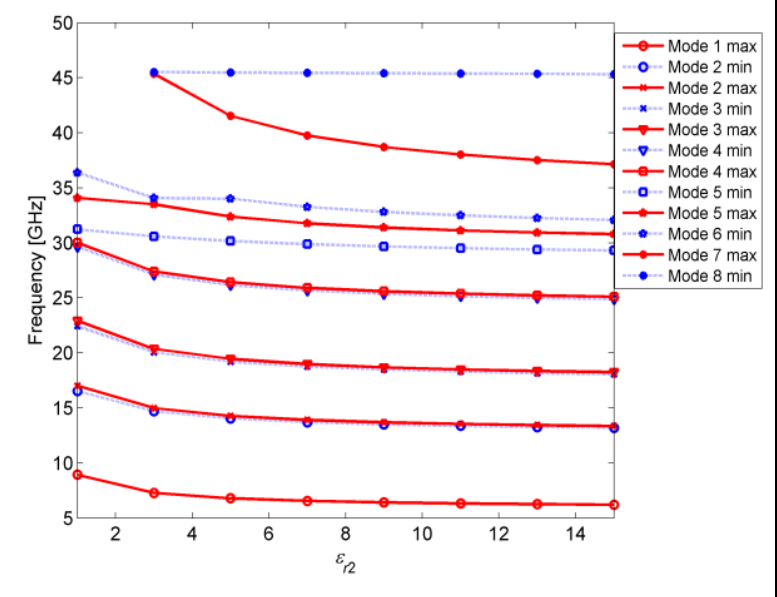

Fig. 5. Frequency limits of the EBGs versus the dielectric constant of the upper dielectric layer for $y$-directed waves

In particular, for parallel-plate noise mitigation, materials with a high dielectric constant allows for obtaining DDs with the fundamental EBG in the low frequency range [21].

Such a feature is required for this kind of applications since the noise spectrum is of the low-pass type [13]. An alternative solution would be to increase the dimensions of the unit cell. However, the dimensions cannot be increased too much due to small dimensions of modern digital and mixed-signal circuits. For filtering out noise with a wide spectrum, the presence of some resonances is not a limiting factor. Therefore, a structure with a DD like in Fig. 2 may be used on a frequency range much wider than the fundamental EBG.

\section{CONCLUSION}

A device built in inhomogeneous strip-line technology, consisting of a patterned surface inserted between two dielectric layers and closed by two metal planes has been considered. The patterned surface has been constructed by a $2 \mathrm{D}$ repetition of a square unit cell, containing a metal patch in the shape of a meander and connected to the lower metal plane through three vias with metal walls, penetrating the lower dielectric layer. The structure has been studied by full-wave electromagnetic simulation. DDs have been calculated in order to find the EBG structure of the device. A large number of EBGs has been shown to exist and a parametric study has been performed in order to provide information for filter design. Existence of resonant modes allows for sensor applications.

\section{ACKNOWLEDGEMENTS}

The research of Ladislau Matekovits has been supported by a Marie Curie International Outgoing Fellowship within the $7^{\text {th }}$ European Community Framework Programme.

\section{REFERENCES}

[1] D. Sievenpiper, L. Zhang, F. J. Boas, N. G.

Yablonovitch, "High-impedance electromagnetic surfaces with a forbidden frequency band", IEEE Trans. Microwave Theory \& Tech., vol. 47, no. 11, pp. 2059-2074, Nov. 1999

[2] D. Sievenpiper, "Forward and backward leaky wave radiation with large effective aperture from an electronically tunable textured surface", IEEE Trans. Antennas Propag., vol. 53, no. 1, pp. 236-247, Jan. 2005

[3] L.-J. Zhang, C.-H. Liang, L. Liang, L. Chen, "A novel design approach for dual-band electromagnetic band-gap structure", Progress in Electromagnetic Research, PIER 4, pp. 81-91, 2008

[4] S. D. Rogers, "Electromagnetic-bandgap layers for broad-band suppression of TEM modes in power planes", Microwave Theory \& Tech., vol. 53, no. 8, pp. 2495-2505, Aug. 2005

[5] T.-L. -L. Wu, Y-H Lin, T.-K. Wang, C.-C. Wang, S.-T. Chen, "Electromagnetic bandgap power/ground planes for wideband suppression of ground bounce noise and radiated emission in high-speed circuits", IEEE Trans. Microwave Theory \& Tech., vol. 53, no. 9, pp. 2935-2942, Sept. 2005

[6] A. De Sabata, L. Matekovits, "Electromagnetic Band-Gap Solution for Mitigation of Parallel-Plate Noise in Power Distribution Networks", Microwave and Optical Technology Letters, vol. 54, no. 7, pp. 1689-1692, 2012

[7] F. de Paulis, L. Raimondo, A. Orlandi, "Impact of shorting vias placement on embedded planar electromagnetic bandgap structures within multilayer printed circuit boards", IEEE Trans. Microwave Theory \& Tech., vol. 58, no. 7, pp. 1867-1876, July 2010

ght propagation in strongly modulated photonic crystals: Refraction like behavior in the vicinity of the Physical Review B, vol. 62, no. 16, Oct. 2000

[9] S. Enoch, G. Tayeb, B. Gralak, "The richness of the dispersion relation of electromag IEEE Trans. Antennas and Propagat., vol.51, no.10, pp.2659-2666, Oct. 2003

[10] A. De Sabata, L. Matekovits, "Unit cell geometry in stripline technology featuring sequential band-gaps between every two consecutive modes", IEEE Antennas and Wireles Propagation Letters, vol. 11, pp. 97-100, 2012

[11]

novel compact broad-band planar filter," IEEE Trans. Microwave Theory Tech., vol. 51, no. 2, pp. 364-369, February 2003

[12] D. Ahn, J.-S. Park, C.-S. Kim, J. Kim, Y. Qian, T.Itoh, "A design of the low-pass filter using the novel microstrip defected ground structure," IEEE Trans. Microwave Theory Tech., vol. 49, no. 1, pp. 86-93, Jan. 2001

[13]

nonuniform photonic band-gap microstripline low-

IEEE Trans. Microwave Theory Tech., vol. 51, no. 2, pp. 564 572, February 2003

[14] C. Gao, Z. N. Chen, Y. Y. Wang, N. Yang, and X. M. Qing, series/parallel

54, no. 4, pp. 1519-1525, April, 2006

[15] Brillouin, Wave Propagation in Periodic Structures, New York: Dover, 1955

[16] Microwave Studio 2009, Computer Simulation Technology

[17] R. Abhari, G. V.Eleftheriades, "Metallo-dielectric electromagnetic band-gap structures for suppression and isolation of the parallelplate noise in high-speed circuits," IEEE Trans. Antennas Propag., vol. 51, no. 6, pp. 1629-1639, June 2003

[18] M.-S. Zhang, Y.-S. Li, C. Jia, L.-P. Li. "A power plane with wideband SSN suppression using a multi-via electromagnetic band-gap structure ", IEEE Microwave Wireless Comp. Letters, vol. 17 , no. 4 , pp. 307-309, April, 2007

[19]

Solution for Switched Parallel Plate Waveguide with Embedded and Applications (JEMWA), in press

Journal of Electromagnetic Waves

[20] M. Schuessler, C. Mandel, M. Puentes, R. Jakobi, "Microwave sensor concepts based on composite right/left-handed metamaterial transmission lines," IEEE Microwave Magazine, vol. 13, no. 2, pp. 57-68, March/April 2012

[21] T. Kamgaing, O. M. Ramahi, "Design and modelling of highimpedance electromagnetic surfaces for switching noise suppression in power planes," IEEE Trans. Electromagn. Compat., vol. 47, no. 3, pp. 479-489, Aug. 2005 